Courrier du Centre International BlaisePascal

38-39 | 2017-2018

Varia

\title{
Le Projet de mandement, ou l'Apocalypse de Pascal
}

\section{Gérard Ferreyrolles}

\section{CpenEdition}

Journals

Édition électronique

URL : https://journals.openedition.org/ccibp/1305

DOI : $10.4000 /$ ccibp. 1305

ISSN : 2493-7460

\section{Éditeur}

Centre international Blaise Pascal

\section{Édition imprimée}

Date de publication : 1 janvier 2019

Pagination : 47-65

ISBN : 978-2-84516-897-8

ISSN : 0249-6674

Référence électronique

Gérard Ferreyrolles, "Le Projet de mandement, ou l'Apocalypse de Pascal », Courrier du Centre International Blaise-Pascal [En ligne], 38-39 | 2017-2018, mis en ligne le 18 juin 2021, consulté le 04 mai 2022. URL : http://journals.openedition.org/ccibp/1305; DOI : https://doi.org/10.4000/ccibp.1305

Ce document a été généré automatiquement le 4 mai 2022.

Centre international Blaise Pascal 


\title{
Le Projet de mandement, ou l'Apocalypse de Pascal
}

\author{
Gérard Ferreyrolles
}

1 Si la campagne consécutive aux Provinciales n'a jusqu'à présent, pour la Lettre d'un avocat au Parlement ou les Écrits des curés, guère retenu l'attention ${ }^{1}$, que faut-il dire du Projet de mandement contre l'" Apologie pour les casuistes", où ce ne sont point un avocat ou de simples curés mais un évêque qui est censé s'exprimer? Pourtant, à la différence de plusieurs pièces de ce corpus, son attribution à Pascal ne soulève aucun doute sérieux et aucune trace de collaboration ne s'y décèle. Le silence est d'autant plus inattendu que l'auteur ne développe nulle part ailleurs dans son œuvre une vision aussi vaste et dramatique de l'histoire de l'Église et du monde. Il est donc temps de réintégrer ce texte étonnant au giron de la critique pascalienne, opération que l'on amorcera ici en s'interrogeant sur son statut générique, puis sur son rapport aux Pensées et son usage de la Bible, enfin sur le sens de l'histoire qui s'y déploie.

\section{I. - Qu'est-ce qu'un mandement ?}

2 Furetière en donne la définition suivante : «Ordre par écrit et rendu public de la part d'une personne qui a autorité et juridiction ${ }^{2}$.» Le mandement n'est donc pas cantonné à la sphère religieuse : Furetière parle de l'« ordonnance d'un juge » comme étant un mandement et il donne cet exemple: « Il y a un mandement de la Ville pour faire des feux de joie ${ }^{3}$.» Mais il est clair que la résonance du terme est d'abord religieuse, et même épiscopale. C'est ce qui ressort des deux premiers exemples de Furetière : «On a publié un mandement de Mr. l'archevêque pour assembler le synode. Un mandement pour l'ouverture du jubilét. » Le Dictionnaire de droit canonique et de pratique bénéficiale de Durand de Maillane, précisera, au XVIII ${ }^{\mathrm{e}}$ siècle, qu'on donne le nom de mandement « aux ordonnances et règlements que font les évêques dans le gouvernement de leurs diocèses $^{5}$ ", et l'auteur ajoute, à propos de l'évêque : «ce droit est essentiellement attaché à l'autorité et à la juridiction ${ }^{6}$ que son caractère lui donne ${ }^{7}$.» Dans l'Église, donc, le droit de publier des mandements n'appartient qu'à l'évêque, sauf à être 
transféré «pendant la vacance du siège aux administrateurs diocésains ${ }^{8}$ ». À ces mandements, les "diocésains doivent se soumettre ${ }^{9}$ ». Même les réguliers qui ont le statut d'exempts ne peuvent refuser de les publier ni se soustraire à leurs interdits ; en matière de foi et de doctrine, ils sont soumis comme les autres - c'est seulement sur l'exécution des mandements touchant les fêtes, les indulgences, les reliques, etc. que peuvent intervenir des aménagements.

Où situer les mandements dans la panoplie des dispositifs d'enseignement de l'évêque ? À côté de la catéchèse et de la prédication, il y a place pour ce qu'on appelle les « actes épiscopaux ». Cette catégorie se subdivise elle-même en trois éléments : 1 . les lettres pastorales: ce sont des lettres "qui contiennent des instructions ou des avis spirituels ${ }^{10}$ » 2. les mandements, qui, eux, renferment des "ordres à exécuter ${ }^{11}$ ", raison pour laquelle ils peuvent prendre la dénomination d'ordonnances ou de règlements ; 3. les circulaires, qui « ne renferment que des avis en matière temporelle ou purement administrative ${ }^{12}$ ». Les "actes épiscopaux» tels qu'ils apparaissent dans la modernité, sous forme de brochures, ont quasiment une date de naissance et une date de mort. Pour leur naissance, «ils sont les enfants de Gutenberg et du concile de Trente $^{13}$ ». De Gutenberg, parce que l'imprimerie permet leur diffusion large et systématique aux diocésains; du concile de Trente, parce qu'il a fait prendre conscience aux évêques de leurs responsabilités pastorales. Durand de Maillane, après avoir indiqué que la publication des mandements était un droit essentiellement attaché au caractère de l'évêque, ajoute que c'est aussi « un devoir que son état lui impose ${ }^{14}$ » et il allègue la session XXV du concile de Trente pour justifier la nécessaire soumission des réguliers aux mandements épiscopaux ${ }^{15}$. Quant à la fin des « actes épiscopaux », elle coïncide, selon Claude Savart ${ }^{16}$, avec la fin du concile Vatican II : la dernière lettre pastorale d'un archevêque de Paris date de 1966. C'est que le concile clôt la période tridentine de l'histoire de l'Église. Désormais, les évêques interviennent par d'autres moyens - les media audiovisuels -, ils le font collectivement - par le biais des conférences épiscopales - et de toute façon leur parole est dominée, pour ne pas dire éclipsée, par la parole pontificale.

4 Durant cette période de quatre cents ans, au sein des "actes épiscopaux ", le mandement apparaît chronologiquement premier. Dans les actes parisiens de la Bibliothèque nationale de France en tout cas, il remonte aux dernières années du $\mathrm{XVI}^{\mathrm{e}}$ siècle et il faut attendre 1697 pour que soit imprimée une «lettre pastorale ». Le mandement semble l'emporter sous l'Ancien Régime, mais au prix d'une dilution de son sens : au départ il s'agit, comme on l'a vu, d'un texte qui renferme des "ordres à exécuter ", mais le mot s'étend ensuite à la partie terminale des lettres pastorales publiées pour le Carême (on comprend pourquoi : cette partie juridique contient les prescriptions relatives à ce temps liturgique de pénitence), puis il peut finir par désigner la totalité de n'importe quelle lettre pastorale. Le mandement continue à prospérer au $\mathrm{XIX}^{\mathrm{e}}$ siècle, mais semble disparaître au $\mathrm{xx}^{\mathrm{e}}$ au profit de la «lettre pastorale ${ }^{17}$ ».

5 Le XvII siècle, lui, vit sous l'empire du mandement ${ }^{18}$. De quoi traite-t-il et comment se présente-t-il ? Deux grands exemples «littéraires" s'offrent: les mandements de Bossuet (il en reste deux) et ceux de Fénelon (il en reste vingt-quatre). Le plus intéressant dans la perspective du Mandement pascalien contre l'« Apologie pour les casuistes» est celui de Bossuet du 3 septembre 1699 qui accompagne la condamnation pontificale de l'Explication des maximes des saints de Fénelon. Bossuet a dû jubiler en le 
rédigeant, car il interdit avec la caution romaine un ouvrage qu'il n'a cessé de combattre : « défendons à toutes personnes de lire ledit livre, même de le garder, sous toutes les peines portées par la constitution [i.e. le Bref pontifical]; enjoignant sous les mêmes peines à ceux qui en auraient quelque exemplaire, de nous les remettre incessamment entre les mains ${ }^{19}$. " Chez Fénelon, la quasi-totalité des mandements se répartissent en deux catégories. La première est celle des mandements qui ordonnent des prières publiques contre les maux de la guerre et de la famine. Deux points communs avec le texte de Pascal : le thème de la colère divine - la main de Dieu « est déjà levée sur nous ${ }^{20}$ ", elle "retarde chaque jour [...] la défaillance prochaine du genre humain $^{21} »-$ et les citations bibliques accordées à ce ton apocalyptique (Fénelon cite en particulier, comme Pascal, Isaïe et les Lamentations de Jérémie). Chez Fénelon comme chez Pascal une lueur d'espérance est néanmoins ménagée. L'autre catégorie de mandements féneloniens est constituée de mandements de Carême, mais le ton est le même que dans la catégorie précédente : «Il semble que voici le temps réservé au feu vengeur pour la fin des siècles ${ }^{22}$ "; Fénelon, comme Pascal et plus encore que lui, décrit une corruption généralisée: "Toute chair a corrompu sa voie; ceux qu'on nomme chrétiens semblent n'en porter le nom que pour l'avilir ${ }^{23}$ ", et Fénelon cite le même chapitre 14 de Jérémie que Pascal sur la désolation du Temple.

Quant à la forme des mandements, elle comporte des éléments mobiles et des éléments fixes. Éléments mobiles : la langue et les destinataires. Les deux vont de pair : quand le texte s'adresse à tous les diocésains, il est rédigé en français; quand il s'adresse uniquement au clergé, il est en principe rédigé en latin. Les éléments fixes sont: 1. L'ouverture, qui comporte de manière stéréotypée les titres du destinateur et la mention des destinataires. Ainsi, chez Fénelon: «François, de par la miséricorde de Dieu et la grâce du Saint-Siège apostolique, archevêque de Cambrai, prince du SaintEmpire, comte du Cambrésis, etc., à tous les fidèles de notre diocèse, salut et bénédiction ${ }^{24}$.» 2. Le corps du texte: l'exposé des motifs qui ont donné lieu au mandement. 3. La conclusion, qui prononce le verdict (par exemple, comme on l'a vu chez Bossuet, l'interdiction de lire tel livre) et expose les moyens de le faire connaître et appliquer (notamment la lecture au prône) : là est employé le verbe mander, qui donne son nom au genre du document et signifie «faire savoir » avec la force d'un ordre.

7 Pour le Projet de mandement pascalien, il est évident qu'il ne peut comporter ni la première partie ni la troisième d'un vrai mandement, car elles relèvent du seul évêque. Pascal fournit, comme plume de l'ombre, le corps du texte, qui, même s'il est assumé et signé par l'évêque, émane couramment, dans l'histoire du genre, du réseau de ses collaborateurs ${ }^{25}$. Il n'est pas difficile de suppléer en quelque façon aux parties initiale et finale manquantes par celles d'un texte très proche, que «les documents anciens attribuent aussi à Pascal ${ }^{26}$ » et que Michel Le Guern donne dans son édition des CEuvres complètes de Pascal, à savoir la censure de l'Apologie pour les casuistes par l'évêque de Nevers le 8 novembre 1658. L'adresse est celle-ci : «Eustache de Chéry, par la grâce de Dieu et autorité apostolique, évêque de Nevers ", à tous les " ecclésiastiques séculiers et réguliers de notre diocèse, salut ${ }^{27}$ ». Après le corps du texte tombe la décision : «Avons fait et faisons très expresses inhibitions et défenses à toutes personnes de notre diocèse, de lire, vendre, acheter ni distribuer ledit livre sous peine d'excommunication ${ }^{28}$. » Enfin, pour la diffusion de cette censure, il est stipulé que «les 
présentes seront lues et publiées » aux prônes "par trois dimanches consécutifs et affichées en la manière accoutumée ${ }^{29}$ ».

\section{II. - Questions d'intra- et d'intertextualité}

II.1. Par intratextualité, j'entends les rapprochements entre le Projet de Mandement et les Pensées. L'abbé Cognet, dans son édition des Provinciales de 1965, n'opère qu'un rapprochement, très général, avec le fragment $800^{30}$. C'est un peu court, mais indéniable. L'argumentation est la même dans les deux textes et repose sur une affirmation centrale, dont deux conséquences sont tirées. L'affirmation est celle-ci : les casuistes corrompus sont de simples particuliers, ils n'engagent pas l'Église, qui consiste proprement dans le corps de sa hiérarchie. Première conséquence de ce découplage : les fidèles n'ont aucune excuse s'ils suivent les décisions des casuistes, car elles ne bénéficient nullement de l'autorité de l'Église. Deuxième conséquence: les infidèles n'ont aucune raison de conclure de la corruption des casuistes à celle de l'Église. L'identité de l'argumentation se traduit par des formulations très proches, voire semblables :

\section{Pensées, fr. 800}

«s'il est vrai, d'une part, que quelques religieux relâchés et quelques casuistes corrompus, qui ne sont point membres de la hiérarchie, ont trempé dans ces corruptions, il est constant, de l'autre, que les véritables pasteurs de l'Église, qui sont les véritables dépositaires de la Parole divine, l'ont conservée immuable contre les efforts de ceux qui ont entrepris de la ruiner [...] l'Église étant proprement dans le corps de la hiérarchie [...] ces hommes [...] sont devenus entre nous ce que les faux prophètes étaient entre les Juifs. »

"les fidèles n'ont aucun prétexte de suivre ces relâchements, qui ne leur sont offerts que par les mains étrangères de ces casuistes, au lieu de la saine doctrine, qui leur est présentée par les mains paternelles de leurs propres pasteurs. »

\section{Projet de mandement}

«si on a sujet de gémir de voir quelques religieux relâchés et quelques casuistes corrompus qui introduisent ces relâchements, on a sujet de bénir Dieu de ce que les pasteurs ordinaires de l'Église leur résistent; et qu'ainsi le corps de la hiérarchie, en quoi consiste proprement l'Église, demeure exempt de ce relâchement, n'y ayant que quelques-unes de ces personnes égarées qui sont hors de la hiérarchie, et qui tiennent entre nous le rang que les faux prophètes tenaient entre les Juifs, qui trempent dans ces impiétés » (p. 573-574).

«ceux qui seraient dans ces erreurs seraient absolument inexcusables de recevoir la fausseté de ces mains étrangères, qui la leur offrent au préjudice de la vérité qui leur est présentée par les mains paternelles de leurs propres pasteurs » (p. 578).

Inutile de prolonger la confrontation : il est clair qu'on a affaire à un même texte dans deux états de sa rédaction, un état avancé au fragment 800 , un état « définitif » dans le Projet de mandement.

Cognet ne propose que ce seul rapprochement, mais les éditeurs précédents, outre celui-ci, en signalent trois autres. Brunschvicg, Boutroux et Gazier, au tome VII (1914) de leur monumentale édition des Æuvres de Pascal, indiquent les fragments 496, 797 et 812. Le premier («Est-il probable que la probabilité assure ? [...]»), sans naturellement 
le contredire, ne présente avec le Projet qu'une zone de superposition trop mince. En revanche, pour les fragments 797 et 812, le doute est impossible :

\section{Pensées, fr. 797}

"Vous ignorez les prophéties, si vous ne savez que tout cela doit arriver : princes, prophètes, pape, et même les prêtres ; et néanmoins l'Église doit subsister.

Par la grâce de Dieu, nous n'en sommes pas là. Malheur à ces prêtres! Mais nous espérons que Dieu nous fera la miséricorde que nous n'en serons point.

1 Saint Pierre, c. 2 [en fait, 2 P 2, 1] : faux prophètes passés, images des futurs. »

\section{Projet de mandement}

«Il est donc indubitable [d'après les Écritures] que ces scandales devaient arriver » (p. 572). «Il n'arrive rien que de conforme à ce que saint Pierre a prédit en cette sorte : "de la même manière qu'il y a eu de faux prophètes entre les Juifs, aussi il s'en élèvera entre vous" [2 P 2, 1] » (p. 574). « Voilà le dernier des malheurs où, par la grâce de Dieu, l'Église n'est pas encore, et où elle ne tombera pas, tant qu'il plaira à Dieu de soutenir ses pasteurs » (p. 575). « "Les princes sont dans la corruption, les prêtres les y accompagnent. Les prophètes les y confirment" [cf. Jr 8, 1]» (p. 578).

\section{Pensées, fr. 812}

«Toute la société entière de leurs casuistes ne peut assurer la conscience dans l'erreur, et c'est pourquoi il est important de choisir de bons guides.

Ainsi ils seront doublement coupables, et pour avoir suivi des voies qu'ils ne devaient pas suivre, et pour avoir ouï des docteurs qu'ils ne devaient pas ouïr. »

\section{Projet de mandement}

«toute la société des casuistes ne saurait assurer la conscience contre la vérité éternelle » (p. 576).

" ceux qui seraient dans ces erreurs seraient [...] doublement coupables dans ces impiétés, et pour avoir reçu des opinions qu'ils ne devaient jamais admettre, et pour les avoir reçues de ceux qu'ils ne devaient point écouter » (p. 578).

11 Michel Le Guern reprend à son compte les trois rapprochements (fr. 800, 797 et 812) signalés par l'édition des Grands Écrivains de la France qu'on vient de mentionner et laisse de côté, non sans raison, le fragment $496^{31}$; il en ajoute un autre, le fragment 698 , qui dénonce, à partir de la Deuxième Épître à Timothée ${ }^{32}$, la recherche par les grands de maîtres qui les flattent dans leurs vices: cette thématique et cette référence paulinienne se retrouvent en effet dans le Projet de mandement, mais les reprises textuelles ne sont pas aussi nettes que dans les cas précédents. Les trois fragments 800 , 797 et 812 suffisent pour nous assurer que le Projet est bien de Pascal - il figure d'ailleurs (sans titre) sous forme de copie dans le fameux recueil 12449 qui contient la Seconde Copie des Pensées ${ }^{33}$. Le Projet a été édité pour la première fois en 1757 (sur la suggestion, hautement significative, de l'abbé d'Étemare et à partir d'une copie provenant de $\mathrm{M}^{\text {lle }}$ de Théméricourt ${ }^{34}$ ), puis par Bossut en 1779. Jean Mesnard accorde un grand poids aux témoignages de Le Roy de Saint-Charles et de Bossut qui affirment même si le P. Guerrier ne l'a pas connu ${ }^{35}$ - qu'il y a bien eu un original de la main de Pascal $^{36}$. Le maître des études pascaliennes n'a aucun doute, qui désigne le Projet de mandement comme un «morceau important de l'œuvre polémique de Pascal ${ }^{37}$ ». J'ajouterai simplement que ce Projet apparaît comme le symétrique du Cinquième Écrit 
des curés de Paris (arrêté le 11 juin 1658), dont l'attribution à Pascal n'est pas contestée. Les deux textes se fondent sur le découplage de la doctrine des casuistes et de celle de l'Église, insistant chacun sur l'une des deux conséquences à en tirer : le Cinquième Écrit développe surtout l'idée que les hérétiques ne sauraient se prévaloir des maximes des casuistes pour accuser de corruption l'Église entière, tandis que le Projet de mandement, par destination, développe surtout l'idée que les fidèles seraient inexcusables de suivre des religieux égarés au lieu de leurs pasteurs hiérarchiquement institués.

II.2. Par intertextualité, j'entends le rapport du Projet à la Bible. Or sur ce point les éditions sont insuffisantes dans leurs références et parfois même silencieuses.

\section{Projet de mandement}

Quelle raison y a-t-il d'être surpris de ce qui arrive dans l'Église, après que JésusChrist « a déclaré que plusieurs y jetteraient le trouble, sous l'apparence néanmoins de la piété, et qu'ils viendraient en son nom pour détourner les hommes de la véritable voie : de sorte que ces désordres, qui croîtraient toujours, seraient enfin si grands dans la fin des siècles, que les élus mêmes en seraient séduits, s'il était possible de les séduire?»(p. 572, sans référence).

\section{La Bible ${ }^{38}$}

"plusieurs viendront sous mon nom, disant: Je suis le Christ; et ils en séduiront plusieurs" (Mt 24, 5) ; «Il s'élèvera un grand nombre de faux prophètes, qui séduiront beaucoup de personnes » $(M t 24,11)$; "il s'élèvera de faux christs et de faux prophètes, qui feront de grands prodiges, et des choses étonnantes, jusqu'à séduire même, s'il était possible, les élus » (Mt 24, 24).

\section{Projet de mandement}

Saint Paul «dit à Timothée qu'il viendrait dans les derniers temps des hommes ayant l'apparence de la piété, mais qui en rejetteraient l'essence; qui seraient pleins d'ambition et d'amour-propre, superbes, calomniateurs, sans amour de Dieu ; qui s'introduiraient dans les maisons des particuliers, et s'assujettiraient les femmes simples, en les flattant dans leurs péchés et dans les désirs de leur cœur ; qui travailleraient sans cesse à devenir savants, et n'arriveraient jamais à la connaissance de la vérité. Et il finit cette peinture en disant qu'ils ne réussiront pas dans leurs desseins, et qu'enfin leur faiblesse et leur impertinence sera connue de tout le monde » (p. 573, avec renvoi à 2 Tm 4, 1-9, ce qui est à la fois trop large et insuffisant).

\section{La Bible}

« il viendra un temps où les hommes ne pourront plus souffrir la saine doctrine; au contraire, ayant une extrême démangeaison d'entendre ce qui les flatte, ils auront recours à une foule de docteurs propres à satisfaire leurs désirs ; et fermant l'oreille à la vérité, ils l'ouvriront à des fables » $(2 \mathrm{Tm} 4,3-4)$; « ils ont été corrompus dans leurs mœurs, semeurs de faux rapports, calomniateurs et ennemis de Dieu. Ils ont été outrageux, superbes, altiers, inventeurs de nouveaux moyens de faire le mal [...] ils n'ont pas compris que ceux qui font ces choses sont dignes de mort (digni sunt morte); et non seulement ceux qui les font, mais aussi quiconque approuve ceux qui les font " (Rm 1, 29-32; "digni sunt » est cité au fr. 698, note en vue du Projet); "dans les dernier jours il viendra des temps fâcheux, car il y aura des hommes amoureux d'eux-mêmes, avares, glorieux, superbes, médisants, [...] qui auront une apparence de piété, mais qui en ruineront la vérité et l'esprit [...], qui s'introduisent 
dans les maisons, et qui traînent après eux comme captives des femmes chargées de péchés, et possédées de diverses passions, lesquelles apprennent toujours, et n'arrivent jamais jusqu'à la connaissance de la vérité [...]. Ce sont des hommes corrompus dans l'esprit, et pervertis dans la foi ; mais le progrès qu'ils feront aura ses bornes ; car leur folie sera connue de tout le monde » $(2 \operatorname{Tm} 3,1-9)$.

Ce ne sont là que quelques exemples. Il faudrait repérer de façon plus rigoureuse les lieux bibliques objets d'allusions, de paraphrases et de citations dans le texte de Pascal. Il faudrait en particulier démêler leur enchevêtrement dans plusieurs séquences du texte et analyser les torsions, les ellipses, les amalgames que Pascal peut imposer aux Écritures. Ce n'est pas là simple souci d'érudition, mais le moyen d'interpréter ce qui fait l'originalité du Projet de mandement, à savoir sa perspective historique, puisqu'elle y est explicitement fondée par Pascal sur des passages scripturaires. Et sur ce plan, les éditeurs ne nous aident guère, qui non seulement sont insuffisants dans leur indexation biblique, mais ne consacrent pas la moindre note à cette vision de l'histoire. Les éditions du Projet de mandement sont un désert herméneutique. Essayons donc d'esquisser une suppléance.

\section{III. - La vision de l'histoire dans le Projet de mandement}

Ce texte est captivant parce qu'il part d'un événement ponctuel pour aboutir à une dramaturgie eschatologique. Le point de départ est la publication de l'Apologie pour les casuistes du P. Pirot, mais le champ s'élargit immédiatement, car derrière la figure singulière de l'auteur, anonyme, de ce livre se masse la foule des casuistes ${ }^{39}$ et derrière cette foule se profile "une Compagnie bien puissante qui soutient ces corruptions » (Projet, p. 573). Il y a péril en la demeure: si les scandales gangrènent le corps de l'Église, c'en est fait d'elle et, indissociablement, de «l'univers» (Projet, p. 574). Et comme Pascal articule cette évolution à celle de l'Ancien Israël - pour lui, le plus ancien peuple de la terre - on est en face d'une vision d'ensemble de l'histoire. De quels modèles Pascal dispose-t-il pour la penser ? On peut en trouver trois :

15 1. Le songe de Nabuchodonosor au deuxième chapitre de Daniel. Il est rapporté au fragment 720 : les différents métaux qui composent la fameuse statue annoncent la succession des empires depuis la prise de Jérusalem par Nabuchodonosor - à savoir l'empire babylonien, puis celui des Mèdes, puis celui des Perses, enfin celui d'Alexandre. Mais ce schéma ne peut intégrer l'histoire de l'Église, puisque la statue s'écroule à l'arrivée du Christ.

2. Le schéma tripartite tiré de saint Paul : règne de la nature, d'Adam à Moïse; règne de la Loi, de Moïse à Jésus ; règne de la grâce, de Jésus à la fin du monde. C'est une scansion communément reçue mais aussi chiffrée, selon le témoignage de Cornelius a Lapide dans le dernier volume de ses Commentarii sur l'Écriture : 2000 ans pour chacune des trois périodes, ce qui place la fin du monde aux alentours de l'an $2000^{40}$. On a un écho de cette tripartition au fragment 754: «La Loi n'a pas détruit la nature, mais elle l'a instruite ; la grâce n'a pas détruit la Loi, mais elle la fait exercer. » Pour autant, Pascal se garde bien de toute spéculation chiffrée et surtout ce schéma ne permet pas de discerner une évolution dans l'histoire de l'Église, comprise en un seul bloc, celui du temps de la grâce. 
17 3. La succession augustinienne des six âges dans le De Genesi contra Manichceos ${ }^{41}$, le sixième âge s'étendant du premier avènement du Christ à son retour glorieux. Pascal se réfère explicitement à ce modèle au fragment 315 : «Les six âges, les six pères des six âges, les six merveilles à l'entrée des six âges, les six orients à l'entrée des six âges. » Mais ce schéma, comme le précédent, ne dessine aucune courbe à l'intérieur du sixième âge et saint Augustin ne se hasarde pas à prophétiser tel événement climatérique dans le temps de l'Église. On en reste au principe général du mélange, sans incidences particulières prévisibles, de la cité terrestre et de la cité de Dieu : «En ce siècle, en ces jours mauvais, depuis la présence corporelle du Christ et de ses apôtres [...] jusqu'à la fin de ce siècle, l'Église de l'exil poursuit sa marche parmi les persécutions du monde et les consolations de Dieu ${ }^{42}$. » Bossuet, dans L'Apocalypse avec une explication, reprochera à saint Augustin, si l'on peut ainsi parler, de présenter de l'histoire de l'Église une vision anhistorique - le combat perpétuel de «deux cités, deux villes, deux empires mêlés selon le corps et séparés selon l'esprit ${ }^{43} »-$, perspective édifiante, certes, mais trop " générale » et « facile ${ }^{44}$ ».

18 Aucun de ces trois schémas n'est opératoire pour Pascal. Sur quoi va-t-il donc se fonder pour donner sens aux événements présents et pour entrevoir l'avenir ? Sur saint Paul ${ }^{45}$, en deux temps : d'abord sur ses prédictions, ensuite sur son principe d'interprétation de l'Écriture. Le premier temps concerne le présent; le second, l'avenir - et leur articulation ne va pas de soi. Pour ce qui est du présent (l'affaire des casuistes), il est comme annoncé dans la Seconde Épître à Timothée et l'Épître aux Romains, que Pascal paraphrase dans le Projet de mandement ${ }^{46}$ en y puisant des critiques pauliniennes contre les philosophes ${ }^{47}$ et les hérétiques qui correspondent à celles que Pascal a émises contre les casuistes dans Les Provinciales, les deux catégories s'amalgamant dans celle des "faux docteurs ${ }^{48}$ ». La superposition est si bien ajustée que Pascal peut conclure : «Qui ne dirait que saint Paul a vu ce qui se passe aujourd'hui à nos yeux? » (Projet, p. 573). La prédiction ou la prophétie paulinienne a aussi l'avantage d'être rassurante, car les séducteurs sont promis à l'échec: Paul finit leur «peinture en disant qu'ils ne réussiront pas dans leurs desseins, et qu'enfin leur faiblesse et leur impertinence sera connue de tout le monde" (ibid.). Pascal considère qu'il en a bien été ainsi dans le différend avec les casuistes corrompus : ils « sont enfin tombés dans des excès qui les ont rendus le sujet de la risée de tout le monde " (ibid.). C'est là un diagnostic d'échec que Pascal a rendu aussi dans la XIV Provinciale (« vos décisions meurtrières sont maintenant en aversion à tout le monde $\left.{ }^{49} »\right)$, dans le Sixième Écrit des curés de Paris en juillet 1658 ( «l'horreur des peuples [...] va directement contre ces excès ${ }^{50} »$ ) et qu'on lit notamment au fragment 571 des Pensées. Ce discrédit des faux docteurs, paradoxal en ce que leurs maximes accommodantes auraient dû au contraire leur attirer les faveurs d'une humanité corrompue, est pour Pascal un signe providentiel : « On ne peut [...] douter que toutes ces choses ne soient conduites par l'ordre de la même Providence qui les a prédites » (Projet, p. 573). On retrouve ici la fin « optimiste » de la XII Provinciale, où Pascal explique que « la violence n'a qu'un cours borné par l'ordre de Dieu, qui en conduit les effets à la gloire de la vérité qu'elle attaque ${ }^{51} »$. Tout semble aller, si l'on ose dire, pour le mieux dans le meilleur des mondes possibles.

19 C'est alors qu'intervient une sorte de renversement. Alors que la victoire semble acquise sur la corruption, Pascal écrit : « nous apprenons par ces mêmes prophéties que ces désordres doivent aller bien plus avant» (Projet, p. 573). S'ouvre alors le second volet d'un diptyque : après le présent, l'avenir. Après un présent où la licence est 
contenue, pour ne pas dire à bout de souffle, un avenir où elle emportera tout. Qu'estce qui peut légitimer ce rebond? Pour Pascal, ce sont les mêmes prophéties qu'il a précédemment convoquées qui le prédisent. Pourtant, les versets de la Seconde Épitre à Timothée qu'il a paraphrasés montrent que les corrompus sont devenus « le sujet de la risée de tout le monde » (ibid.). Une expression directement traduite de saint Paul peut aider à dépasser l'aporie : les faux docteurs doivent venir "dans les derniers temps ${ }^{52}$ ", expression qui peut désigner ces temps qui sont les derniers - c'est-à-dire le sixième âge du monde, celui où la Révélation est devenue complète et définitive en JésusChrist - mais aussi la fin du monde. On est renvoyé par là au modèle des prophéties énoncées par le Christ, qui sont à double détente : annonce de la ruine de Jérusalem et annonce de la destruction du monde. De fait, Pascal cite $^{53}$ en les juxtaposant deux versets évangéliques qui prédisent, l'un, la ruine de Jérusalem - « Malheur à celles qui seront enceintes en ce jour-là » (Mt 24, 19) - et l'autre, la fin du monde - « croyez-vous qu'alors le fils de l'homme trouve de la foi sur la terre? » (Lc 18, 8). On pourrait même dire que Pascal télescope les deux versets en articulant le second au premier par un « alors » de son invention. La corruption présente des casuistes dans l'Église serait donc une image et une étape de la corruption généralisée de cette institution dans l'avenir.

Comment Pascal représente-t-il ce processus? Selon un principe paulinien qui, on le sait, gouverne l'exégèse pascalienne: "hœec autem omnia in figura contingebant illis » $(1$ Co 10,11$)$ - ce que Sacy résume en tête de chapitre dans sa traduction par: "Juifs, figure des chrétiens ", et ce que Pascal transpose dans le Projet de mandement par la formule : « la Synagogue a été l'image de l'Église» (p. 574). L'histoire de la seconde va donc répéter celle de la première, qui se termine mal. Cette double histoire est très (trop ?) clairement structurée dans le texte du Projet, qui distingue la «source», le « progrès » et enfin « la consommation de l'impiété » (ibid.). La source, ce sont les faux prophètes ; le progrès connait deux moments - la contagion des grands, puis celle des peuples - ; la consommation correspond à la corruption des prêtres : « la mesure étant ainsi comblée, la fin de l'Église et de l'univers doit arriver avec la seconde venue du Messie» (ibid.). Mais Dieu n'aura-t-il pas pitié ? La réponse de Pascal est terrible : «il n'y a plus de miséricorde à attendre, parce qu'il n'y a plus personne pour la demander » (Projet, p. 575).

21 Ce schéma pose plusieurs questions :

22 1. Est-il repérable dans les citations que Pascal fait de l'Ancien Testament? Pas avec autant de netteté. Jérémie dit bien que l'abomination est née des prophètes ${ }^{54}$, mais pour les étapes suivantes, on n'aperçoit pas de chronologie marquée dans la corruption des grands, des peuples et des prêtres. Par exemple, au chapitre 5 de Jérémie, il est écrit : "Les prophètes ont annoncé de fausses doctrines de la part de Dieu: les prêtres y ont donné les mains, et mon peuple y a pris plaisir ${ }^{55}$. " Les prêtres semblent les premiers contaminés, en tout cas pas les derniers, et les grands sont absents. Pascal a opéré une sorte de rationalisation dans le traitement des citations pour en tirer une succession peut-être forcée.

23 2. Pascal a-t-il construit ou hérité ce schéma? Avant d'affirmer qu'il l'a construit, il faudrait s'assurer de l'absence de sources - ce qui n'est pas encore acquis. Saint-Cyran, convaincu d'une décadence de l'Église, pourrait être une piste. Sur un point précis du schéma, on peut avancer une réponse : Pascal soutient que « la destruction de l'ancien Temple et de la Synagogue » (Projet, p. 574) au moment du premier avènement du Messie est arrivée dans le temps de la plus grande décadence de la Synagogue. Où a-t-il 
trouvé ce thème ? Saint Augustin est peu disert, quoique affirmatif, dans La Cité de Dieu : «du jour où elle n'eut plus de prophètes, la nation juive, c'est certain, entra en décadence (deterior facta est) ${ }^{56}$. » Bossuet sera beaucoup plus prolixe, en 1681, dans la deuxième partie du Discours sur l'histoire universelle ${ }^{57}$. Il s'appuie sur le chapitre 11 de Zacharie, que Pascal cite une fois (au fragment 736) mais à propos de la prophétie des trente deniers. Bossuet se fonde aussi sur Flavius Josèphe, mais dans un domaine principalement politique - les «brouilleries survenues dans la maison des Asmonéen $\mathbf{s}^{58} »$. Je proposerai pour ma part un passage du Talmud que Pascal a trouvé dans le Pugio fidei et qu'il transcrit au fragment 718 des Pensées: "C'est une tradition entre nous [les Juifs] que, quand le Messie arrivera, la Maison de Dieu destinée à la dispensation de sa parole sera pleine d'ordure et d'impureté, et que la sagesse des scribes sera corrompue et pourrie. Ceux qui craindront de pécher seront réprouvés du peuple et traités de fous et d'insensés. »

24 3. Pourquoi Pascal rend-il concomitantes «la fin de l'Église » et celle de «l'univers »? Dans cette coïncidence se dévoile la finalité de l'histoire et donc son sens ultime, qui est de construire la Jérusalem céleste, c'est-à-dire d'enfanter les élus qui la peupleront. L'histoire s'arrêtera quand le nombre des élus sera complet. Comme c'est la mission de l'Église que de les enfanter, quand elle aura achevé sa tâche, le monde n'aura plus de raison d'être. Il faut relire là-dessus la Prière pour [...] le bon usage des maladies : « Ô Dieu, qui ne laissez subsister le monde et toutes les choses du monde que pour exercer vos élus, et pour punir les pécheurs ${ }^{59}$ !» L'histoire des royaumes humains est ordonnée, qu'ils en aient ou non conscience, à l'édification d'un royaume qui n'est pas de ce monde. D'où une quatrième question :

4. Comment l'Église remplira-t-elle sa mission si elle est totalement corrompue ? Faut-il entendre que sa fin - son terme - sera une destruction punitive s'abattant sur celle qui a failli? C'est ce qu'affirme la conclusion du Projet de mandement, qui parle du «consentement général dans l'erreur qui doit attirer le dernier jugement de Dieu » (p. 578). Mais alors Pascal a-t-il oublié le début de Projet, où il énonçait que Dieu avait " assuré [l'Église] d'une possession éternelle de la vérité » (p.572) ? La résolution de cette difficulté peut venir d'un petit mot qui se trouve, lui, au milieu du texte : la fin de l'Église doit arriver, y est-il écrit, au temps où les fidèles et les prêtres auront «presque tous consenti aux impiétés des faux docteurs» (p. 574). C'est la théologie du "petit reste d'Israël » que l'on trouve en contexte eschatologique dans un passage d'Isaïe que Pascal traduit au fragment 735 : « Le Seigneur dit ainsi : Pour l'amour de mes serviteurs, je ne perdrai pas tout Israël, mais j'en réserverai quelques-uns ${ }^{60}$. $" A u$ reste, les maux dont souffre présentement l'Église n'annoncent pas une imminente fin du monde: "Voilà le dernier des malheurs où, par la grâce de Dieu, l'Église n'est pas encore " (Projet, p. 575). Qu'est-ce qui retient le bras de Dieu? La fidélité des pasteurs. L'existence même du Projet de mandement prouve la fidélité de l'épiscopat, comme l'existence des Écrits des curés prouve la fidélité du clergé de second ordre. Mais qu'estce qui garantit cette fidélité ? La grâce de Dieu obtenue par la prière des fidèles. Nous voyons donc que la situation n'est pas désespérée : dans la contagion corruptrice qui s'étend graduellement des casuistes aux grands, au peuple et aux prêtres, les casuistes sont gangrenés à coup sûr, les grands ne valent pas mieux (le fragment 698, qui est une note préparatoire au Projet de mandement, les juge "abandonnés à l'esprit du mensonge »), mais les prêtres résistent, et leur résistance durera aussi longtemps que le peuple chrétien demandera « à Dieu la continuation [de leur] zèle » (Projet, p. 575). Telle était déjà l'exhortation au mois de septembre 1656, en plein combat contre les 
casuistes, de la première lettre à Charlotte de Roannez : «il est bien juste que la prière soit continuelle, quand le péril est continuel ${ }^{61}$. » Nous ne sommes donc pas à la dernière extrémité, mais au milieu du gué.

Pour terminer et permettre de situer la démarche pascalienne dans le Projet de mandement, j'esquisserai deux comparaisons avec des démarches qui peuvent paraître similaires.

La première confrontation est avec un ouvrage paru en 1657, L'Ancienne Nouveauté de l'Écriture sainte, ou l'Église triomphante en terre par Charpy de Sainte-Croix, qui prédit à la lumière de l'Apocalypse le triomphe temporel de l'Église sur toutes les nations après la défaite de l'Antéchrist. On relèvera déjà une différence massive, mais que son évidence même peut faire oublier, à savoir que pour dessiner le futur de l'Église et du monde Pascal, paradoxalement, ne fait dans le Projet aucune référence à l'Apocalypse. C'est qu'il craint le piège de ce que le fragment 478 appelle les «extravagances des apocalyptiques et préadamites, millénaires, etc. ", piège dans lequel Charpy de SainteCroix tombe allègrement en prédisant que Jésus-Christ viendra exercer sa domination sur la terre en la personne d'un roi de la race de David qui sera son lieutenant général. Charpy soutient même que cette prédiction se réalisera avant la fin du XvII ${ }^{e}$ siècle. Point n'est besoin de s'appesantir sur le fait que Pascal, naturellement, n'avance aucune date quant à la réalisation de son scénario pour l'avenir. Plus intéressante, la règle herméneutique qu'Arnauld formule en 1657 dans ses Remarques sur les principales erreurs d'un livre intitulé "L'Ancienne Nouveauté de l'Écriture sainte, ou l'Église triomphante en $t^{t} r e^{62} »$. Cette règle entend à la fois justifier les interprétations figuratives de la Bible par les Pères et écarter les spéculations arbitraires comme celles de Charpy : "Quand les deux termes d'un rapport sont vrais en eux-mêmes, en sorte que personne n'en puisse douter, et qu'il n'y a que l'application qui soit nouvelle, on n'y peut guère trouver à redire ${ }^{63}$ » : ainsi le rapport de figuration établi par les Pères entre Jésus retrouvé dans le Temple le troisième jour et sa résurrection dans le même délai. On peut dire que le schéma pascalien satisfait à la règle posée car le premier avènement de Jésus-Christ au moment de la plus grande corruption de la Synagogue est regardé comme un fait certain et que son second avènement au moment où la foi sera le plus compromise dans l'Église ne l'est pas moins («lorsque le fils de l'homme viendra, pensez-vous qu'il trouve de la foi sur la terre $\left.?^{64} »\right)$ - outre que le rapport de figuration entre la Synagogue et l'Église est lui-même établi sur l'Écriture et les Pères.

La seconde comparaison sera entre la démarche de Pascal et celle des figuristes du xvIII ${ }^{e}$ siècle. On ne saurait oublier que la première publication du Projet, selon une source autorisée ${ }^{65}$, fut faite à l'initiative du chef de file des figuristes, l'abbé d'étemare, en 1757 : il devait y trouver une caution pour sa propre lecture prophétique de la Bible. Et de fait, plusieurs spécialistes font remonter au xvII ${ }^{e}$ siècle les prodromes du mouvement qu'il incarne : Saint-Cyran, écrit Hervé Savon, apprend à Port-Royal à lire la préfiguration de son histoire "dans les moindres détails du texte sacré66"; pour Catherine Maire, Duguet, le créateur du figurisme, est «directement inspiré ${ }^{67}$ » par Bossuet, Sacy et Pascal. Ces deux historiens citent la même phrase de Sacy dans $L a$ Genèse traduite en français: "dans tout ce qui lui arrive [le peuple juif] est la figure vivante et animée de tout ce qui devait arriver à Jésus-Christ et à son Église ${ }^{68}$. » N'est-ce pas la conviction qui sous-tend le Projet de mandement : l'histoire de l'Église répète celle de la Synagogue - même décadence, même fin programmée? Cependant, il faut marquer les différences. De façon anecdotique, on relèvera que si un figuriste a poussé 
à la publication du Projet, c'est un antifiguriste, l'abbé de Bonnaire, qui a fait rééditer en 1735 les Remarques d'Arnauld contre le livre de Charpy de Sainte-Croix. Plus profondément, des écarts apparaissent: d'Étemare interprète l'histoire présente en construisant des analogies avec des figures bibliques - il assimile par exemple les PortRoyalistes aux Maccabées - ; Pascal, dans le Projet de Mandement, ne produit pas de luimême des analogies toujours récusables pour arbitraire, mais s'en tient à des figures validées par le Nouveau Testament (en l'occurrence saint Paul sur l'Église et la Synagogue) et appuie son interprétation sur des prophéties et prédictions scripturaires. Et ce qui retient Pascal au moins autant que le présent de l'Église, c'est son avenir, discerné en termes spirituels et eschatologiques, alors que les figuristes reportent l'accomplissement des Écritures «dans un futur temporel et politique ${ }^{69}$ ». Il faudrait, pour faire avancer l'enquête, examiner ce qu'avance d'Étemare dans son Parallèle de l'histoire du peuple d'Israël et de l'histoire de l'Église ${ }^{70}$. Mais des points de divergence sont d'ores et déjà notables: d'Étemare interprète l'histoire profane à partir des figures bibliques $^{71}$; le " petit reste » des fidèles est identifié par lui à Port-Royal détruit et à ses fidèles persécutés, alors que dans le texte pascalien, même si les casuistes constituent une foule, le peuple et ses légitimes pasteurs en résistance victorieuse forment le grand nombre : le "petit reste» est placé à la fin de l'histoire. Quand il écrit le Projet de mandement, Pascal est par position du côté d'une hiérarchie exempte de la corruption des casuistes : pour lui, ni les prêtres ni les évêques ne sont touchés (en tout cas, ils ne le sont pas encore) par l'expansion du mystère d'iniquité. Pascal fait plus qu'adhérer à l'Église visible, il en porte la parole, alors que les figuristes s'en détachent (elle n'est plus pour eux qu'une "simple écorce extérieure ${ }^{72} »$ ), à l'applaudissement des protestants, au point qu'ils tendent à remplacer le corps de la hiérarchie comme lieu de la légitimité par le corps des fidèles ${ }^{73}$. On pourrait toutefois supputer que si Pascal avait vécu la destruction du monastère de Port-Royal et la promulgation de la Bulle Unigenitus il eût modifié sa perspective malgré tout optimiste sur le présent de l'Église. En ce sens, l'abbé d'Étemare représenterait un Pascal possible, un Pascal convaincu par les événements que la corruption a gagné le cœur de l'Église. Mais il est un point essentiel au figurisme qui est totalement absent, comme Philippe Sellier l'a $v^{74}$, du Projet de mandement, c'est la conversion d'Israël. La décadence de l'Église, pour les figuristes, est le signe que bientôt Israël viendra revivifier les chrétiens de la gentilité : "le temps des Juifs n'est pas éloigné ", écrit Duguet ${ }^{75}$, qui dissocie, contre l'exégèse traditionnelle, la conversion des Juifs prédite dans l'Épître aux Romains de l'Apocalypse. L'Israël du Projet de mandement n'est que l'Israël ancien, celui de la Synagogue détruite, et nullement l'espoir de reviviscence d'une Église exténuée.

Par rapport à Charpy de Sainte-Croix comme par rapport aux figuristes, on mesure la prudence de Pascal, qui ne donne aucune date pour les événements prédits ni ne se lance dans des spéculations fondées sur de supposées révélations particulières. Touchant les prophéties sur l'avenir de l'Église et du monde, il garde, tout en restant saisissant, la distance de celui qui écrit au fragment 751 : «on n'entend les prophéties que quand on voit les choses arrivées ", la distance aussi, fondée sur la parole du Christ («Ce n'est pas à vous de savoir les temps et les moments que le Père a réservés à son souverain pouvoir $\left.{ }^{76} »\right)$, que saint Augustin ne cesse d'opposer aux exégèses contournées de son collègue Hesychius ${ }^{77}$. Mais une fois définies les bornes entre lesquelles se situe le Projet de mandement, il reste assurément à progresser encore dans l'intelligence de son contenu. 


\section{NOTES}

1. À l'exception notamment de J. Mesnard, «La collaboration des écrivains de PortRoyal aux censures des évêques contre l'Apologie pour les casuistes (1658-1659)» (Chroniques de Port-Royal, $\mathrm{n}^{\circ}$ 32, 1983, p. 3-20), et d'O. Jouslin, "Rien ne nous plait que le combat». La campagne des "Provinciales» de Pascal. Étude d'un dialogue polémique (Clermont-Ferrand, Presses Universitaires Blaise Pascal, 2007, 2 vol.).

2. A. Furetière, Dictionnaire universel, La Haye et Rotterdam, Arnout et Reinier Leers, 1690. Article « Mandement ».

3. Ibid.

4. Ibid.

5. P. T. Durand de Maillane, Dictionnaire de droit canonique et de pratique bénéficiale, Paris, J.-B. Bauche, 1761. Article « Mandements ».

6. Ce sont les deux termes qu'employait Furetière.

7. Durand de Maillane, art. cit.

8. Abbé J.-H.-R. Prompsault, Dictionnaire raisonné de droit et de jurisprudence en matière civile ecclésiastique, Paris, Migne, 1849. Article « Mandements ».

9. Durand de Maillane, art. cit.

10. Prompsault, op. cit., article « Lettres pastorales ».

11. Ibid. Autre différence, de forme cette fois, avec les lettres pastorales: celles-ci peuvent être fort longues, alors que les mandements sont relativement brefs.

12. Ibid.

13. Cl. Savart, "Deux siècles d'enseignement épiscopal. Les lettres pastorales des archevêques de Paris, 1802-1966 ", Revue d'histoire de l'Église de France, vol. 86, n 216, 2000, p. 121.

14. Durand de Maillane, art. cit.

15. Voir ibid.

16. Voir art. cit., p. 121. L'analyse de l'auteur, fondée sur l'étude des actes épiscopaux du seul diocèse de Paris, n'est pas nécessairement extensible à tous les diocèses. Le cardinal Barbarin, par exemple, a publié ces dernières années plusieurs «lettres pastorales » dans son diocèse de Lyon (information communiquée par L. Thirouin).

17. Un exemple pris au hasard (et qui appellerait donc confirmation) : dans les archives de l'évêché de Luçon, consultables sur internet, on constate qu'en 1898 et 1899 les textes publiés par l'évêque sont intitulés « lettre pastorale et mandement " (en un seul titre) tandis qu'à partir de 1900 et jusqu'au dernier document numérisé, on ne trouve plus que des «lettres pastorales».

18. D'autant que les évêques ne semblent pas lésiner en la matière. Dans sa thèse sur $L a$ Réforme pastorale en France au XVII siècle (Desclée de Brouwer, 1956, 2 vol.), P. Broutin cite ce constat de Mgr Le Camus, évêque de Grenoble de 1671 à 1707 et proche de PortRoyal : « La maladie des évêques est de faire beaucoup d'ordonnances [le terme, comme celui de règlement, est synonyme de mandement] et de ne guère s'appliquer à les faire ordonner » (t. I, p. 244), et il intitule significativement une sous-partie du chapitre qu'il 
consacre à Claude Joly, évêque d'Agen de 1665 à 1678: «Ordonnances, ordonnances, ordonnances» (t. I, p. 275).

19. Bossuet, Cuvres complètes [désormais : OCB], Paris, Bloud et Barral, 1865, 12 vol., t. VI, p. 263.

20. Fénelon, Euvres complètes, Paris (J. Leroux et Jouby), Lille et Besançon, 1848-1852, 10 vol., t. VI, p. 178. Mandement du 20 avril 1709.

21. Ibid. Ce qui est souligné, ici et dans les autres citations, l'est par moi.

22. Ibid., p. 180. Mandement du 24 février 1710.

23. Ibid.

24. Ibid., p. 161. Mandement du 15 novembre 1701.

25. À quel évêque ce Projet, qui n'a jamais abouti, était-il destiné ? À François Faure, évêque d'Amiens? J. Mesnard penche plutôt pour l'hypothèse d'un écrit « rédigé sans qu'aucune destination précise lui ait été réservée, simplement pour servir à l'occasion " (art. cit., p. 16). Il ajoute que Pascal a élaboré d'autres textes pour des censures épiscopales contre l'Apologie: les fragments 771 et 811 (voir ibid., p. 16-17). Rappelons que ces censures - une vingtaine au total - se sont échelonnées d'avril 1658 à mai 1659 (avec une exception: celle de l'évêque de Soissons, publiée en octobre 1659), le jugement du tribunal de l'Inquisition romaine qui condamna l'Apologie le 21 août 1659 rendant inutile la poursuite du mouvement. La date de rédaction du Projet de mandement n'est pas connue, mais elle est vraisemblablement antérieure au mois de novembre 1658: Pascal sera absorbé alors par les affaires de la roulette, puis par l'impression (de décembre 1658 à février 1659) des Lettres de A. Dettonville et enfin empêché par la maladie.

26. J. Mesnard, Euvres complètes de Pascal [désormais: OC], Paris, Desclée de Brouwer, 1964-1992, 4 vol. parus, t. I, p. 40.

27. Censure d'un livre anonyme intitulé "Apologie pour les casuistes, etc.» faite par Mgr l'illustrissime et révérendissime évêque de Nevers, dans Euvres complètes de Pascal, éd. M. Le Guern, Paris, Gallimard, « Bibliothèque de la Pléiade », t. I, 1998, p. 942.

28. Ibid., p. 944.

29. Ibid.

30. Les Pensées sont citées, ici et dans tout le reste de cette étude, dans l'édition de Ph. Sellier et G. Ferreyrolles, Paris, Librairie Générale Française, "Le Livre de Poche classique ", 2000. Les Écrits des curés de Paris et le Projet de mandement sont cités dans l'édition de L. Cognet, mise à jour par G. Ferreyrolles, des Provinciales, Paris, Éditions Classiques Garnier, 2010.

31. En tout cela, il s'accorde avec J. Mesnard, art. cit., p. 20 (note 16).

32. $2 \operatorname{Tm} 4,3$.

33. Voir OC, t. I, p. 295.

34. Voir OC, t. I, p. 974 et 260.

35. Son Premier Recueil recopie la copie du recueil $12449\left(\mathrm{RC}^{2}\right)$.

36. Voir $O C$, t. I, p. 974 et 260.

37. OC, t. I, p. 260. Attribution réitérée par J. Mesnard, art. cit., p. 16-19.

38. La Bible, trad. Sacy, éd. Ph. Sellier, Paris, Robert Laffont, « Bouquins », 1990. 
39. Le Projet de mandement parle de leur «nombre étrange» (p.571), c'est-à-dire extraordinaire.

40. Voir J.-R. Armogathe, L'Antéchrist à l'âge classique. Exégèse et politique, Paris, Mille et une nuits, 2005, p. 207-221.

41. I, 23, 35.

42. La Cité de Dieu, XVIII, 51, 2, trad. G.Combès, Paris, Desclée de Brouwer, «Bibliothèque Augustinienne ", vol. 36, 1960.

43. L'Apocalypse avec une explication, Préface, IV, dans OCB, t. II, p. 163.

44. Ibid. Bossuet, lui, distingue dans un «Abrégé de l'Apocalypse » situé à la fin de son ouvrage trois temps dans l'histoire de l'Église: celui des persécutions, au commencement; puis les mille ans - chiffre symbolique par lequel il faut entendre quasiment «tout le temps que le monde dure» ("Abrégé de l'Apocalypse », XXXIV, dans $O C B$, t. II, p. 286) - de règne de l'Église, où elle est à couvert des persécutions universelles; enfin, "la dernière tentation de l'Église » (ibid., XXXI, dans OCB, t. II, p. 285) : Satan sera déchaîné pour un court temps et vaincu à jamais.

45. Mais pas sur sa tripartition nature/Loi/grâce.

46. P. 573. Il s'agit de $2 \operatorname{Tm} 4,3-4 ; \operatorname{Rm~1,~29-32;~} 2 \operatorname{Tm} 3$, 1-9. Les textes sont cités cidessus, section II. 2.

47. C'est eux qui sont visés, indique Sacy en tête de sa traduction de Rm 1.

48. Projet, p. 576, en écho aux magistri de 2 Tm 4, 3.

49. Éd. cit., p. 386.

50. Éd. cit., p. 557.

51. Éd. cit., p. 348.

52. Projet, p. $573 ; 2 \mathrm{Tm} 3,1$ : « in novissimis diebus ».

53. Voir Projet, p. 574.

54. Voir Jr 23, 15 ; cité dans le Projet, p. 574-575.

55. Jr 5, 31 ; cité dans le Projet, p. 575.

56. La Cité de Dieu, trad. cit., XVIII, 45, 1.

57. Voir les chapitres 17, 18 et 19. Le chapitre 19 commence ainsi : " Dans ce déclin de la religion et des affaires des Juifs, à la fin du règne d'Hérode, et dans le temps que les pharisiens introduisaient tant d'abus, Jésus-Christ est envoyé sur la terre » $(O C B, \mathrm{t}$. IX, p. 462-462).

58. Discours sur l'histoire universelle, II, 18, dans OCB, t. IX, p. 461.

59. Prière pour [...] le bon usage des maladies, III, dans OC, t. IV, p. 999.

60. Is 65,8 .

61. Lettres à Melle de Roannez, lettre 1, OC, t. III, p. 1030.

62. L'ouvrage ne sera publié qu'en 1665, mais Nicole nous apprend dans son « Avis au lecteur " qu'il avait connu auparavant une diffusion considérable sous forme de copies (voir A. Arnauld, CEuvres, Paris-Lausanne, S. d'Arnay, 1775-1783, t. V, p. 323).

63. Remarques, éd. cit., p. 355.

64. Lc 18, 8. Traduction Sacy.

65. Le Roy de Saint-Charles (voir OC, t. I, p. 974). 
66. H. Savon, "Le figurisme et la "Tradition des Pères" ", dans Le Grand Siècle et la Bible, J.-R. Armogathe (dir.), Paris, Beauchesne, 1989, p. 762.

67. C. Maire, De la cause de Dieu à la cause de la Nation. Le jansénisme au XVIII siècle, Paris, Gallimard, 1998, p. 169.

68. Cité par H. Savon, art. cit., p. 767, et C. Maire, op. cit., p. 168.

69. H. Savon, art. cit., p. 785. L'adjectif « temporel» ne peut manquer de faire songer à Charpy.

70. Ouvrage écrit en 1712, mais publié en 1723, s.l. Au demeurant, il existe des figuristes, comme l'abbé d'Asfeld, qui n'acceptent pas de considérer Israël comme figure de l'Église.

71. Voir C. Maire, op. cit., p. 183.

72. Ibid., p. 197.

73. Voir ibid., chap. VIII («L'ecclésiologie de la résistance »), p. 205-234.

74. Voir Pascal et saint Augustin, Paris, Albin Michel, 1995, p. 481-482.

75. Explication du mystère de la Passion de Notre-Seigneur Jésus-Christ suivant la Concorde (Paris, 1728), cité par C. Maire, op. cit., p. 174.

76. Ac 1, 7. Traduction Sacy.

77. Voir saint Augustin, Epist. 199-80, De fine sceculi (Patrologia Latina 33, 904-925).

INDEX

Mots-clés : mandement, apocalypse, apologie, intertextualité

Keywords : pastoral letter, apocalypse, apology, intertextuality

\section{AUTEUR}

GÉRARD FERREYROLLES

Professeur de littérature française à l'Université Paris-Sorbonne 